\title{
Uniqueness of solutions to the helically reduced wave equation with Sommerfeld boundary conditions
}

\author{
C. G. Torre ${ }^{a)}$ \\ Department of Physics, Utah State University, Logan, Utah 84322-4415
}

(Received 28 March 2006; accepted 12 May 2006; published online 10 July 2006)

\begin{abstract}
We consider the helical reduction of the wave equation with an arbitrary source on $(n+1)$-dimensional Minkowski space, $n \geqslant 2$. The reduced equation is of mixed elliptic-hyperbolic type on $\mathbf{R}^{n}$. We obtain a uniqueness theorem for solutions on a domain consisting of an $n$-dimensional ball $B$ centered on the reduction of the axis of helical symmetry and satisfying ingoing or outgoing Sommerfeld conditions on $\partial B \approx S^{n-1}$. Nonlinear generalizations of such boundary value problems (with $n=3$ ) arise in the intermediate phase of binary inspiral in general relativity. (C) 2006 American Institute of Physics. [DOI: 10.1063/1.2212667]
\end{abstract}

\section{INTRODUCTION}

Recent approaches to the quasistationary approximation of the intermediate phase of binary inspiral in general relativity have led to the consideration of reductions of the Einstein equations by a helical Killing vector field (see Refs. 1-3 and references therein). To date, model problems have been analyzed consisting of helical reductions of linear and nonlinear wave equations in $(3+1)$-dimensional Minkowski space-time with various sources using Sommerfeld conditions on a spherical boundary. ${ }^{1}$ These helically reduced equations have the challenging feature of being of mixed elliptic-hyperbolic type on their three-dimensional domain. More precisely, they are elliptic in an inner cylindrical region surrounding the sources and hyperbolic outside this cylindrical region. There appear to be no general theorems to handle existence and uniqueness of solutions to partial differential equations of mixed type. Results tend to be specific to individual equations or limited classes of equations, and even then the equations which have been most studied are defined in two dimensions. ${ }^{4}$ From the investigations of Ref. 1 it appears that the boundary value problem arising from helical reduction of (linear and nonlinear) wave equations using Sommerfeld conditions on an exterior boundary is well-posed. Solutions have been constructed and appear to be unique. This is somewhat remarkable since the boundary intersects both the hyperbolic and elliptic domains. In particular, one might not expect a single (Sommerfeld) condition on a closed boundary to enforce uniqueness of solutions. ${ }^{1}$

Some light was shed on this issue by the work of Ref. 5, where the helical reduction of the $(2+1)$-dimensional wave equation was shown to define a symmetric-positive system on an annular region in $\mathbf{R}^{2}$ such that the Sommerfeld boundary value problem was well-posed-solutions exist and, in particular, are unique. Unfortunately, it is not known how to generalize these results (i.e., symmetric positivity of the reduced equation) to higher dimensions. Moreover, the helical reduction of the $(2+1)$-dimensional wave equation leads to a boundary value problem on a twodimensional region with an outer circular boundary which need never intersect the circle of degeneracy of the symbol of the reduced partial differential equation. In higher dimensions, the spherical outer boundary necessarily intersects the "light cylinder" where the symbol is degenerate so the boundary conditions must be imposed both in the elliptic and in the hyperbolic regions. (Unless, of course, the boundary is completely contained in the elliptic region, which is not of physical interest and which, in any case, leads to a standard elliptic boundary value problem). This

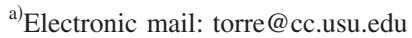


makes the problem qualitatively different in the physical $(3+1)$ space-time dimensions (and in higher dimensions).

Thus, it is of interest both from mathematical physics and gravitational physics viewpoints to better understand the nature of boundary value problems arising from helical reduction of wave equations. Here we shall provide a uniqueness theorem for the helical reduction of the $(n+1)$-dimensional wave equation with arbitrary sources and with Sommerfeld boundary conditions. The proof is remarkably elementary and employs an approach used by Protter to study a generalization of the Tricomi problem. ${ }^{6}$

\section{THE HELICALLY REDUCED WAVE EQUATION}

We will be considering the helical reduction of the wave equation with an arbitrary source on $(n+1)$-dimensional Minkowski space, with $n \geqslant 2$. The space-time manifold is $N=\mathbf{R}^{n+1}$ with metric

$$
\eta=-d t \otimes d t+d x \otimes d x+d y \otimes d y+\delta_{i j} d z^{i} \otimes d z^{j},
$$

where Latin indices $i, j=1,2, \ldots, n-2$. The wave equation for $\Psi: N \rightarrow \mathbf{R}$ with a prescribed source $F: N \rightarrow \mathbf{R}$ is

$$
-\Psi_{t t}+\Psi_{x x}+\Psi_{y y}+\delta^{i j} \Psi_{i j}=F .
$$

Note we use the notation where subscripts on a function indicate partial derivatives. The helical reduction is accomplished by assuming the source and solutions are invariant with respect to the isometry group $(G)$ generated by

$$
K=\partial_{t}+\Omega\left(x \partial_{y}-y \partial_{x}\right), \quad \Omega=\text { const } .,
$$

which is equivalent to

$$
\mathcal{L}_{K} F=\mathcal{L}_{K} \Psi=0 .
$$

In cylindrical coordinates $\left(t, \rho, \phi, z^{i}\right)$, the metric and Killing vector field are

$$
\begin{gathered}
\eta=-d t \otimes d t+d \rho \otimes d \rho+\rho^{2} d \phi \otimes d \phi+\delta_{i j} d z^{i} \otimes d z^{j}, \\
K=\partial_{t}+\Omega \partial_{\phi},
\end{gathered}
$$

the wave equation is

$$
-\Psi_{t t}+\frac{1}{\rho} \partial_{\rho}\left(\rho \Psi_{\rho}\right)+\frac{1}{\rho^{2}} \Psi_{\phi \phi}+\delta^{i j} \Psi_{i j}=F,
$$

and the invariance condition (2.4) is

$$
\Psi_{t}=-\Omega \Psi_{\phi}, \quad F_{t}=-\Omega F_{\phi} .
$$

Introducing $\varphi=\phi-\Omega t$, (2.8) means there exists functions $u$ and $f$ such that

$$
\Psi\left(t, \rho, \phi, z^{i}\right)=u\left(\rho, \varphi, z^{i}\right), \quad F\left(t, \rho, \phi, z^{i}\right)=f\left(\rho, \varphi, z^{i}\right) .
$$

We then get the reduced equation defining helically invariant solutions to (2.2)

$$
\frac{1}{\rho} \partial_{\rho}\left(\rho u_{\rho}\right)+\frac{\chi(\rho)}{\rho^{2}} u_{\varphi \varphi}+\delta^{i j} u_{i j}=f,
$$

where

$$
\chi(\rho)=1-\Omega^{2} \rho^{2} .
$$


The locus of points where $\chi(\rho)=0$ is the "light cylinder". Inside the light cylinder ( $\rho$ $<1 / \Omega)$ Eq. (2.10) is elliptic and outside the light cylinder $(\rho>1 / \Omega)$ Eq. (2.10) is hyperbolic.

A useful geometric interpretation of this reduction is as follows. The set of orbits of the group generated by $K$ defines a manifold $M=N / G \approx \mathbf{R}^{n}$. The functions $\left(\rho, \varphi, z^{i}\right)$ are $G$-invariant and define cylindrical coordinates on $M$. In these coordinates the projection $\pi: N \rightarrow M$ is simply

$$
\pi\left(t, x, y, z^{i}\right)=\left(\rho, \varphi, z^{i}\right)
$$

and satisfies $\pi_{*} K=0$. The $G$-invariant functions $F$ and $\Psi$ on $N$ correspond to functions $f$ and $u$ on $M$, respectively, via

$$
F=\pi^{*} f, \quad \Psi=\pi^{*} u .
$$

The inverse metric on $N$ is given by

$$
\eta^{\sharp}=-\partial_{t} \otimes \partial_{t}+\partial_{\rho} \otimes \partial_{\rho}+\frac{1}{\rho^{2}} \partial_{\phi} \otimes \partial_{\phi}+\delta^{i j} \partial_{i} \otimes \partial_{j} .
$$

Being $G$-invariant, $\eta^{\sharp}$ projects to a tensor field $q$ on $M$. Using (2.12),

$$
q=\pi_{*} \eta^{\sharp}=\partial_{\rho} \otimes \partial_{\rho}+\frac{\chi(\rho)}{\rho^{2}} \partial_{\varphi} \otimes \partial_{\varphi}+\delta^{i j} \partial_{i} \otimes \partial_{j} .
$$

This tensor field is well-defined everywhere on $M$, but it does not determine a metric on $M$ because $q$ has no inverse on the light cylinder. While the metric on $N$ does not induce a metric on $M$, the metric volume form $\epsilon$ on $N$ does define a volume form $\nu$ on $M$ as follows. Define

$$
\omega=K \rightarrow \epsilon,
$$

which satisfies

$$
L_{K} \omega=0, \quad K \rightarrow \omega=0 .
$$

Consequently, $\omega$ is the pull-back by $\pi$ of a volume form $\nu$ on $M$. It is easy to check that

$$
\nu=\rho d \rho \wedge d \varphi \wedge d z^{1} \wedge \cdots \wedge d z^{n-2} .
$$

The volume form $\nu$ defines a scalar density of weight $1, \sigma=\rho$, on $M$.

We will use Greek indices to label tensor fields on $M$. Introduce a torsion-free derivative operator $\nabla_{\alpha}$. The reduced equation (2.10) is equivalent to

$$
\frac{1}{\sigma} \nabla_{\alpha}\left(\sigma q^{\alpha \beta} \nabla_{\beta} u\right)=f
$$

To see this, we first note that because of the density weights (2.19) is in fact independent of the choice of torsion-free derivative $\nabla_{\alpha}$. Using the cylindrical coordinate derivative operator, $\nabla_{\alpha}=\partial_{\alpha}$, in (2.19) we obtain (2.10). For what follows we rewrite (2.19) as

$$
\nabla_{\alpha}\left(h^{\alpha \beta} \nabla_{\beta} u\right)=\partial_{\alpha}\left(h^{\alpha \beta} u_{\beta}\right)=\tilde{f},
$$

where $\tilde{f}=\sigma f$ is a scalar density of weight 1 and $h^{\alpha \beta}=\sigma q^{\alpha \beta}$ is a tensor density of weight 1 given by

$$
h^{\rho \rho}=\rho, \quad h^{i j}=\rho \delta^{i j}, \quad h^{\varphi \varphi}=\frac{1}{\rho} \chi=\frac{1}{\rho}-\Omega^{2} \rho .
$$




\section{ENERGY INTEGRAL}

The key ingredient in our uniqueness theorem is the following generalized energy integral. Fix a domain $B \subset M$ and define

$$
E[u]=\int_{B}\left[\left(a u+b^{\gamma} u_{\gamma}\right) \partial_{\alpha}\left(h^{\alpha \beta} u_{\beta}\right)\right],
$$

where $a$ and $b^{\alpha} \partial_{\alpha}$ are a function and vector field to be specified later. The integrand involving $a$ can be written as

$$
a u \partial_{\alpha}\left(h^{\alpha \beta} u_{\beta}\right)=\frac{1}{2} \partial_{\alpha}\left(h^{\alpha \beta} a_{\beta}\right) u^{2}-a h^{\alpha \beta} u_{\alpha} u_{\beta}+\partial_{\alpha}\left[a u h^{\alpha \beta} u_{\beta}-\frac{1}{2} h^{\alpha \beta} a_{\beta} u^{2}\right] .
$$

The integrand involving $b^{\gamma}$ can be written as

$$
b^{\gamma} u_{\gamma} \partial_{\alpha}\left(h^{\alpha \beta} u_{\beta}\right)=\frac{1}{2} \partial_{\gamma}\left(h^{\alpha \beta} b^{\gamma}\right) u_{\alpha} u_{\beta}-b_{, \alpha}^{\gamma} h^{\alpha \beta} u_{\gamma} u_{\beta}+\partial_{\alpha}\left[b^{\gamma} u_{\gamma} h^{\alpha \beta} u_{\beta}-\frac{1}{2} b^{\alpha} h^{\beta \gamma} u_{\gamma} u_{\beta}\right] .
$$

Again, while these expressions use the coordinate derivative, they are in fact independent of the choice of torsion-free derivative operator. The divergences integrate to the boundary and we have

$$
\begin{aligned}
E[u]= & \int_{B}\left\{\frac{1}{2} \partial_{\alpha}\left(h^{\alpha \beta} a_{\beta}\right) u^{2}-a h^{\alpha \beta} u_{\alpha} u_{\beta}+\frac{1}{2} \partial_{\gamma}\left(h^{\alpha \beta} b^{\gamma}\right) u_{\alpha} u_{\beta}-b_{, \alpha}^{\gamma} h^{\alpha \beta} u_{\gamma} u_{\beta}\right\} \\
& +\int_{\partial B} n_{\alpha}\left\{\left(a u+b^{\gamma} u_{\gamma}\right) h^{\alpha \beta} u_{\beta}-\frac{1}{2} h^{\alpha \beta} a_{\beta} u^{2}-\frac{1}{2} b^{\alpha} h^{\beta \gamma} u_{\gamma} u_{\beta}\right\} .
\end{aligned}
$$

If there were a metric on $B, n_{\alpha}$ could be defined in terms of the unit normal to the boundary and the metric-induced volume element of the boundary. Without a metric $n_{\alpha}$ is still defined, of course, but its definition is necessarily more involved. We give the definition in the Appendix.

\section{UNIQUENESS THEOREM}

We are now ready to formulate the boundary value problem of interest. We consider solutions to Eq. (2.20) on a ball of radius $R$,

$$
B=\left\{\left(\rho, \varphi, z^{i}\right) \mid 0 \leqslant \rho^{2}+\delta_{i j} z^{i} z^{j} \leqslant R^{2}\right\} .
$$

The boundary $\partial B$ is the sphere $S^{n-1}$ of radius $R$. Using (A7), we have in spherical coordinates $\left(r, \theta_{1}, \ldots, \theta_{n-1}\right)$,

$$
n_{\alpha} d x^{\alpha}=d r
$$

We impose Sommerfeld-type conditions on $\partial B$ of the type used in Ref. 1. These take the form (in cylindrical coordinates)

$$
\frac{1}{R}\left(\rho u_{\rho}+z^{i} u_{i}\right) \pm \Omega \partial_{\varphi} u=\tau, \quad \text { on } \partial B,
$$

where we have included a function $\tau: \mathbf{S}^{n} \rightarrow \mathbf{R}$ to allow for nonradiative (e.g., monopole) contributions to $u$ (see Remark (ii) below). These boundary conditions can be understood as follows. Sommerfeld conditions for $\Psi$ are, strictly speaking, decay conditions on $\Psi_{r} \mp \Psi_{t}$ at spatial infinity in $N$, where $r$ denotes the spatial radius at fixed $t$ in $N$ (see, e.g., Ref. 7 for a discussion of Sommerfeld conditions). These conditions select ingoing/outgoing radiation. At a finite radiusnecessary for numerical computations-one imposes conditions of the form 


$$
\Psi_{r} \mp \Psi_{t}=\tau, \quad \text { on } \partial B .
$$

Applying the helical symmetry reduction the conditions (4.4) reduce to (4.3).

Remark: (i) If $R>1 / \Omega$ the boundary passes through both the elliptic and hyperbolic domains. (ii) The functions $\tau$ and $\tilde{f}$ cannot be specified independently. Using (4.3) the integral of (2.20) over $B$ implies

$$
\int_{B} \tilde{f}=\int_{\partial B} \sigma \tau
$$

Since (2.20) and the boundary conditions (4.3) only involve derivatives of $u$, solutions to these equations can only be unique up to an additive constant. In fact, this is the only freedom in the solution. Our main result is the following.

Theorem: Given $\Omega, \tilde{f}: B \rightarrow \mathbf{R}$, and $\tau: \partial B \rightarrow \mathbf{R}$, any two solutions to (2.20) on B with boundary conditions (4.3) differ at most by a constant.

Proof: Consider the difference of two solutions, $u=u_{1}-u_{2} ; u$ satisfies (2.20) and (4.3) with $\tilde{f}=0$ and $\tau=0$, respectively. Consequently, $E[u]=0$ for any choices of the function $a$ and vector field $b=b^{\alpha} \partial_{\alpha}$. We choose these as

$$
a=-1, \quad b=\frac{2}{1-n}\left[\rho \partial_{\rho}+z^{i} \partial_{i} \pm R \Omega \partial_{\varphi}\right] .
$$

Note that

$$
b^{\alpha} u_{\alpha}=0, \quad \text { on } \partial B .
$$

A straightforward computation, using (4.3) with $\tau=0$ in the boundary integral, then gives

$$
\begin{aligned}
0= & \int_{B}\left\{\left(\frac{1}{n-1}\right) \sigma\left[\left(u_{\rho}^{2}+\delta^{i j} u_{i} u_{j}\right)+\left(\frac{1}{\rho^{2}}+\Omega^{2}\right) u_{\varphi}^{2}\right]\right\}+\int_{\partial B}\left(\frac{1}{n-1}\right) \sigma \frac{R}{\rho^{2}}\left\{\left(z^{i} z^{j}+\rho^{2} \delta^{i j}\right) u_{i} u_{j}+(1\right. \\
& \left.\left.+\Omega^{2} \delta_{i j} z^{i} z^{j}\right) u_{\varphi}^{2} \pm 2 R \Omega z^{i} u_{i} u_{\varphi}\right\} .
\end{aligned}
$$

The volume integrand (in the first integral) is manifestly non-negative for $n \geqslant 2$. We now show that the boundary integrand (in the second integral) is also non-negative.

We first note that the boundary integrand is invariant under orthogonal transformations of the $z^{i}$. Thus, given any point $\left(\rho, \varphi, z^{i}\right)$, we can rotate the $z^{i}$ axes such that $z^{i}=(z, 0,0, \ldots, 0)$, where $z^{2}=\delta_{i j} z^{i} z^{j}$. The boundary integrand at the given point is then

$$
\begin{aligned}
& \left(\frac{1}{n-1}\right) \sigma \frac{R}{\rho^{2}}\left\{\left(z^{i} z^{j}+\rho^{2} \delta^{i j}\right) u_{i} u_{j}+\left(1+\Omega^{2} \delta_{i j} z^{i} z^{j}\right) u_{\varphi}^{2} \pm 2 R \Omega z^{i} u_{i} u_{\varphi}\right\} \\
& \quad=\left(\frac{1}{n-1}\right) \sigma \frac{R}{\rho^{2}}\left\{z^{2} u_{1}^{2}+\rho^{2} \delta^{i j} u_{i} u_{j}+\left(1+\Omega^{2} z^{2}\right) u_{\varphi}^{2} \pm 2 R \Omega z u_{1} u_{\varphi}\right\} \\
& \quad \geqslant\left(\frac{1}{n-1}\right) \sigma \frac{R}{\rho^{2}}\left\{u_{\varphi}^{2}+\left(R u_{1} \pm \Omega z u_{\varphi}\right)^{2}\right\} \geqslant 0 .
\end{aligned}
$$

Because both integrands in (4.8) are non-negative they must each vanish. From the volume integrand it follows immediately that

$$
u_{\alpha}=0
$$




\section{ACKNOWLEDGMENT}

This work was supported in part by National Science Foundation Grant PHY-0244765 to Utah State University.

\section{APPENDIX: THE DIVERGENCE THEOREM WITHOUT A METRIC}

Consider an $n$-dimensional orientable manifold $M$, a torsion-free derivative operator $\nabla_{\alpha}$ on $M$, and a vector density of weight $1, V^{\alpha}$. Given $B \subset M$, Stokes theorem implies an identity of the form

$$
\int_{B} \nabla_{\alpha} V^{\alpha}=\int_{\partial B} n_{\alpha} V^{\alpha}
$$

Normally this divergence theorem is proved using a metric on $M$. However, this is not necessary. Here, we shall give a version of the divergence theorem and, in particular, give a formula for $n_{\alpha}$ without using a metric.

The manifold $M$, being orientable, comes equipped with a nowhere vanishing $n$-form density of weight minus 1 , denoted by $\eta_{\alpha_{1}, \ldots, \alpha_{n}}$, and a totally antisymmetric contravariant tensor density of weight $1, \widetilde{\eta}^{\alpha_{1}, \ldots, \alpha_{n}}$, such that

$$
\tilde{\eta}^{\alpha_{1}, \ldots, \alpha_{n}} \eta_{\beta_{1}, \ldots, \beta_{n}}=n ! \delta_{\beta_{1}}^{\left[\alpha_{1}\right.} \cdots \delta_{\beta_{n}}^{\left.\alpha_{n}\right]} .
$$

Both $\eta_{\alpha_{1}, \ldots, \alpha_{n}}$ and $\widetilde{\eta}^{\alpha_{1}, \ldots, \alpha_{n}}$ are constant for any choice of $\nabla_{u}$.

The boundary $\partial B$ is an oriented submanifold in $M$ embedded by $i: S \rightarrow M$, i.e., $\partial B=i(S) . S$ is equipped with an $(n-1)$-form density of weight minus $1, \xi_{a_{1}, \ldots, a_{n-1}}$, and a skew, contravariant rank $(n-1)$ tensor density of weight $1, \widetilde{\xi}^{a_{1}, \ldots, a_{n-1}}$, satisfying

$$
\widetilde{\xi}^{a_{1}, \ldots, a_{n-1}} \xi_{b_{1}, \ldots, b_{n-1}}=(n-1) ! \delta_{b_{1}}^{\left[a_{1}\right.} \cdots \delta_{b_{n-1}}^{\left.a_{n-1}\right]} .
$$

(In this appendix only we use Latin indices to denote tensors on $S$.)

To apply Stokes theorem we define an $(n-1)$-form

$$
\omega_{\alpha_{1}, \ldots, \alpha_{n-1}}=V^{\beta} \eta_{\beta \alpha_{1}, \ldots, \alpha_{n-1}} .
$$

We then have (using differential form notation)

$$
\int_{B} \nabla_{\alpha} V^{\alpha}=\int_{B} d \omega=\int_{\partial B} \omega=\int_{S} \frac{1}{(n-1) !} \xi^{a_{1}, \ldots, a_{n-1}\left(i^{*} \omega\right)_{a_{1}, \ldots, a_{n-1}} .}
$$

Now, at points of $\partial B$ we can write

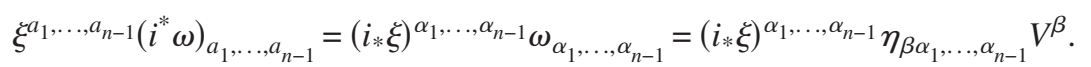

Thus we have

$$
n_{\beta}=\frac{1}{(n-1) !}\left(i_{*} \xi\right)^{\alpha_{1}, \ldots, \alpha_{n-1}} \eta_{\beta \alpha_{1}, \ldots, \alpha_{n-1}} .
$$

An alternative approach to the integral over $B$ in (A1) is to note that it is independent of the choice of $\nabla_{\alpha}$. If we fix a Riemannian metric $g_{\alpha \beta}$ on $M$, and use the metric-compatible derivative operator, we have available the more traditional form of the divergence theorem,

$$
\int_{B} \nabla_{\alpha} V^{\alpha}=\int_{\partial B} \sqrt{\gamma} \hat{n}_{\alpha} W^{\alpha},
$$

where 


$$
W^{\alpha}=\frac{1}{\sqrt{g}} V^{\alpha},
$$

$\hat{n}_{\alpha}$ is the outwardly oriented unit normal to $\partial B$, and $\sqrt{\gamma}$ is the induced volume element on $\partial B$. The result (A8) is, of course, equivalent to the manifestly metric independent result (A5) above, as can be verified by using the identity

$$
\hat{n}_{\beta}=\frac{1}{(n-1) !} \frac{\sqrt{g}}{\sqrt{\gamma}}\left(i_{*} \xi\right)^{\alpha_{1}, \ldots, \alpha_{n-1}} \eta_{\beta \alpha_{1}, \ldots, \alpha_{n-1}}
$$

${ }^{1}$ J. Whelan, W. Krivan, and R. Price, Class. Quantum Grav. 17, 4895 (2000); J. Whelan, C. Beetle, W. Landry, and R. Price, ibid. 19, 1285 (2002); Z. Andrade et al., Phys. Rev. D 70, 064001 (2004); B. Bromley, R. Owen, and R. Price, gr-qc/0502121 (2005); C. Beetle, B. Bromley, and R. Price, gr-qc/0602027 (2006).

${ }^{2}$ C. Klein, Phys. Rev. D 70, 124026 (2004).

${ }^{3}$ J. Friedman and K. Uryu, Phys. Rev. D 73, 104039 (2006).

${ }^{4}$ J. Rassias, Lecture Notes on Mixed Type Partial Differential Equations (World Scientific, Singapore, 1990).

${ }^{5}$ C. G. Torre, J. Math. Phys. 44, 6223 (2003).

${ }^{6}$ M. Protter, Indiana Univ. Math. J. 3, 435 (1954).

${ }^{7}$ R. Courant and D. Hilbert, Methods of Mathematical Physics (Interscience, New York, 1962), Vol. 2. 